\title{
Spectral nuclear properties of NLS1 galaxies ${ }^{\star}$
}

\author{
E. O. Schmidt ${ }^{1, \star \star}$, D. Ferreiro ${ }^{1,2}$, L. Vega Neme ${ }^{1,2}$, and G. A. Oio ${ }^{1}$ \\ 1 Instituto de Astronomía Teórica y Experimental (IATE), Universidad Nacional de Córdoba, CONICET, \\ Observatorio Astronómico de Córdoba, Laprida 854, Córdoba, Argentina \\ e-mail: eduschmidt@oac.unc.edu.ar \\ 2 Observatorio Astronómico, Universidad Nacional de Córdoba, Laprida 854, 5000 Córdoba, Argentina
}

Received 19 July 2016 / Accepted 4 August 2016

\begin{abstract}
Context. It is not yet well known whether narrow line Seyfert 1 (NLS1) galaxies follow the $M_{\mathrm{BH}}-\sigma_{\star}$ relation found for normal galaxies. Emission lines, such as [SII] or [OIII] $\lambda 5007$, have been used as a surrogate of the stellar velocity dispersion and various results have been obtained. On the other hand, some active galactic nuclei (AGNs) have shown Balmer emission with an additional intermediate component (IC) besides the well-known narrow and broad lines. The properties of this IC have not yet been fully studied. Aims. In order to re-examine the location of NLS1 in the $M_{\mathrm{BH}}-\sigma_{\star}$ relation, we test some emission lines, such as the narrow component (NC) of $\mathrm{H} \alpha$ and the forbidden [NII] $\lambda \lambda 6548,6584$ and [SII] $\lambda \lambda 6716,6731$ lines, as replacements for $\sigma_{\star}$. On the other hand, we study the properties of the IC of $\mathrm{H} \alpha$ found in 14 galaxies of the sample to find a link between this component, the central engine, and the remaining lines. We also compare the emission among the broad component (BC) of $\mathrm{H} \alpha$ and those emitted at the narrow line region (NLR) to detect differences in the ionizing source in each emitting region.

Methods. We have obtained and studied medium-resolution spectra $\left(170 \mathrm{~km} \mathrm{~s}^{-1}\right.$ FWHM at $\left.\mathrm{H} \alpha\right)$ of $36 \mathrm{NLS} 1$ galaxies in the optical range $\sim 5800-6800 \AA$. We performed a Gaussian decomposition of the $\mathrm{H} \alpha+[\mathrm{NII}] \lambda \lambda 6548,6584$ profile to study the distinct components of $\mathrm{H} \alpha$ and $[\mathrm{NII}]$ lines. We also measured the [SII] lines.

Results. We obtained black hole $(\mathrm{BH})$ masses in the range $\log \left(M_{\mathrm{BH}} / M_{\odot}\right)=5.4-7.5$ for our sample. We found that, in general, most of the galaxies lie below the $M_{\mathrm{BH}}-\sigma_{\star}$ relation when the NC of $\mathrm{H} \alpha$ and [NII] lines are used as a surrogate of $\sigma_{\star}$. The objects are closer to the relation when [SII] lines are used. Nevertheless, the galaxies are still below this relation and we do not see a clear correlation between the $\mathrm{BH}$ masses and $\mathrm{FWHM}_{[\mathrm{SII}]}$. Besides this, we found that 13 galaxies show an IC, most of which are in the velocity range $\sim 700-1500 \mathrm{~km} \mathrm{~s}^{-1}$. This is same range as in AGN types and is well correlated with the FWHM of BC and, therefore, with the $\mathrm{BH}$ mass. On the other hand, we found that there is a correlation between the luminosity of the $\mathrm{BC}$ of $\mathrm{H} \alpha$ and $\mathrm{NC}$, IC, [NII] $\lambda 6584$, and [SII] lines.
\end{abstract}

Key words. galaxies: active - galaxies: Seyfert - galaxies: nuclei - galaxies: kinematics and dynamics

\section{Introduction}

Narrow line Seyfert 1 galaxies are a subclass of active galactic nuclei (AGNs). The most common defining criterion for these objects is the width of the broad component of their optical Balmer emission lines in combination with the relative weakness of the [OIII] $\lambda 5007$ emission with full width half maximum $(\mathrm{FWHM}) \leq 2000 \mathrm{~km} \mathrm{~s}^{-1}$ and the relative weakness of [OIII] $\lambda 5007 / \mathrm{H} \beta \leq 3$ (Osterbrock \& Pogge 1985; Goodrich 1989). Optical spectroscopy classifications suggest that NLS1 represent about $15 \%$ of the whole population of Seyfert 1 galaxies (Williams et al. 2002).

Observations of NLS1, as well as other AGNs, clearly show different types of emission lines in their spectra. Depending on the width of the emitted lines, they are classified as narrow lines with FWHM of a few hundred of $\mathrm{km} \mathrm{s}^{-1}$, or broad lines, with

\footnotetext{
$\star$ The reduced spectra as FITS files are only available at the CDS via anonymous ftp to cdsarc.u-strasbg. $f r(130.79 .128 .5)$ or via http://cdsarc.u-strasbg.fr/viz-bin/qcat?]/A+A/596/A95

$\star \star$ Visiting Astronomer, Complejo Astronómico El Leoncito operated under agreement between the Consejo Nacional de Investigaciones Científicas y Técnicas de la República Argentina and the National Universities of La Plata, Córdoba, and San Juan.
}

FWHM of a few thousand $\mathrm{km} \mathrm{s}^{-1}$. This implies two different regions of line formation, i.e, narrow line region (NLR) and the broad line region (BLR), respectively.

In the middle of the '90s various works on QSOs suggested that the traditional broad line region (BLR) consists of two components: one component with a FWHM of $\sim 2000 \mathrm{~km} \mathrm{~s}^{-1}$, called the intermediate line region (ILR), and another very broad component (VBLR) with a FWHM of $\sim 7000 \mathrm{~km} \mathrm{~s}^{-1}$ blueshifted by $\geq 1000 \mathrm{~km} \mathrm{~s}^{-1}$ (Brotherton et al. 1994; Corbin \& Francis 1994). Brotherton suggested that the intermediate line region (ILR) arises in a region inner to the narrow line region (NLR). Mason et al. (1996) examined the profiles of $\mathrm{H} \alpha, \mathrm{H} \beta$, and [OIII] lines and found evidence for an intermediate-velocity $\left(F W H M \leq 1000 \mathrm{~km} \mathrm{~s}^{-1}\right)$, line-emitting region that produces significant amounts of both permitted and forbidden lines. Crenshaw \& Kraemer (2007) identified a line emission component with width $F W H M=1170 \mathrm{~km} \mathrm{~s}^{-1}$ for the Sy1 NGC 4151, most probably originating between the BLR and NLR. Hu et al. (2008) reported evidence for an intermediate line region in a sample of 568 quasar selected from the Sloan Digital Sky Survey (SDSS). They investigated the $\mathrm{H} \beta$ and FeII emission lines and observed that the conventional broad $\mathrm{H} \beta$ emission line could be decomposed into two components: one component with a very 
broad FWHM and the another with an intermediate FWHM. This ILR, whose kinematics seems to be dominated by infall, could be located in the outskirts of the BLR. In addition, Crenshaw et al. (2009) detected ILR emission with width of $680 \mathrm{~km} \mathrm{~s}^{-1}$ in the spectrum of NGC 5548. Mao et al. (2010) studied a sample of 211 narrow line Seyfert 1 galaxies selected from the SDSS, finding that the $\mathrm{H} \beta$ profile can be fitted well by three (narrow, intermediate, and broad) Gaussian components with a ratio $F W H M_{\mathrm{BC}} / F W H M_{\mathrm{IC}} \sim 3$ for the entire sample. Also, they suggested that the intermediate components originate from the inner edge of the torus, which is scaled by dust $K$-band reverberation. Related to this, there are some questions about the ILR. Is it linked to the central engine of the AGN? How is it related to the BLR emission? One of the aims of this paper is to answer these questions studying the intermediate component of $\mathrm{H} \alpha$ in NLS1 galaxies.

On the other hand, and related to the central dynamics, according to several authors there is a correlation between the $\mathrm{BH}$ masses and the velocity dispersion of the stars of the bulge of the host galaxy (e.g., Ferrarese \& Merritt 2000; Gebhardt et al. 2000; Tremaine et al. 2002). Because of the difficulty of measuring the stellar velocity dispersion in AGNs, sometimes emission lines are used as a surrogate. For example, in the case of the [OIII] $\lambda 5007$ line, various authors found that NLS1 are off the $M_{\mathrm{BH}}-\sigma_{\star}$ relation (e.g., Mathur et al. 2001; Bian \& Zhao 2004; Grupe \& Mathur 2004; Mathur \& Grupe 2005a,b; Zhou et al. 2006) and on the contrary, other researchers found that these galaxies are on the relation (Wang \& Lu 2001; Botte et al. 2005). Komossa \& Xu (2007) found that using [SII] lines as a replacement for the stellar velocity dispersion, NLS1 galaxies are on the relation. In this paper we re-examine the location of the NLS1 galaxies in the $M_{\mathrm{BH}}-\sigma_{\star}$ relation using the narrow component of $\mathrm{H} \alpha$ (originated in the NLR) and the forbidden emission lines [NII] and [SII] as surrogates of stellar velocity dispersion.

As a whole, NLS1 galaxies represent a very interesting class of objects, and it is important to study them both from their structural and dynamical point of view and investigate how these objects fit into the unification schemes for AGNs. In order to study the intermediate component of $\mathrm{H} \alpha$ and to test various emission lines as a replacement for $\sigma_{\star}$ we studied 36 NLS1 galaxies, of which 27 are in the Southern Hemisphere. We carried out a spectroscopic study of these galaxies to analyze their internal kinematics and to derive properties regarding their BLR, NLR, and ILR. In Sect. 2 we present the sample and observations; a description of how the measurements were carried out is detailed in Sect. 3; in Sect. 4 we present the results regarding the nuclear dynamics, for example, the $\mathrm{BH}$ masses of the sample, location of the galaxies in the $M_{\mathrm{BH}}-\sigma_{\star}$ relation, and the intermediate component of $\mathrm{H} \alpha$; and in Sect. 5 we show how the luminosities of the different lines are related. We present some curious individual cases in Sect. 6 and in Sect. 7 we draw our conclusions. Throughout this paper, we use the cosmological parameters $H_{0}=70 \mathrm{~km} \mathrm{~s}^{-1} \mathrm{Mpc}^{-1}, \Omega_{\mathrm{M}}=0.3$, and $\Omega_{\Lambda}=0.7$.

\section{Observational data}

\subsection{Sample and observations}

We have selected NLS1 galaxies from the Véron \& Véron catalog (Véron-Cetty \& Véron 2010) with redshifts $z<0.15$ and brighter than $m_{\mathrm{b}}<18$ with $\delta \leq 10^{\circ}$. Of those objects, we chose galaxies whose nuclear kinematics are poorly or even not studied. This way our sample consists of 36 galaxies, of which 27 are in the Southern Hemisphere, while the remaining have $0^{\circ}<\delta \leq 10^{\circ}$. This coordinate range allows us to observe the sample from the Complejo AStronómico el LEOncito (CASLEO), in Argentina.

Observations were performed in different campaigns between 2011 and 2014. We carried out long-slit spectroscopy using the REOSC spectrograph at CASLEO $2.15 \mathrm{~m}$ RitcheyChrétien telescope, San Juan, Argentina. The spectrograph has a Tektronix $1024 \times 1024 \mathrm{CCD}$ attached with $24 \mu \mathrm{m}$ pixels. The galaxies were observed using a 2.7 arcsec wide slit and the extractions of each spectrum were of $\sim 2.3$ arcsec. For a mean distance of $240 \mathrm{Mpc}$ (mean redshift of $\sim 0.06$ ) the spectra correspond to the central $\sim 3 \mathrm{kpc}$ in projected distance. We used a 600 line $\mathrm{mm}^{-1}$ grating giving a resolution of $170 \mathrm{~km} \mathrm{~s}^{-1}$ FWHM around $\mathrm{H} \alpha$. The observations cover the spectral range from $5800 \AA$ to $6800 \AA$. The spectra were calibrated in wavelengths using comparison lines from a $\mathrm{Cu}-\mathrm{Ne}-\mathrm{Ar}$ lamp and three standard stars were observed each night to flux calibrate the spectra. Standard data reduction techniques were used to process the data, mainly ccdred and longslit packages included in IRAF $^{1}$. The obtained spectra have a mean signal to noise of $\sim 16$ around $6000 \AA$. Although some galaxies in our sample already have spectra, in most cases they have a lower resolution or are not flux calibrated (as in the cases of $2 \mathrm{df}$ and $6 \mathrm{df}$ spectra). In our sample, 11 objects have SDSS spectra with a very similar resolution. They were taken with the SDSS spectrograph, which has a fiber diameter of 3 arcsec. Table A.1 lists the galaxy name, right ascension, declination, apparent $B$ magnitude, redshift, date of the observation, and exposure time.

\subsection{NLS1 spectra}

Here we present the spectra of 36 NLS1s for the red spectral region 5800-6800 $\AA$. In this work we focus on this spectral region to study in detail the emission lines such as $\mathrm{H} \alpha+[\mathrm{NII}] \lambda \lambda 65486584$ and [SII] $\lambda \lambda 67166731$.

The beginning of the spectral region presented conforms to the higher initial wavelength for the red observations, while there were usually sky absorption lines near $7000 \AA$. This made the measurement of sulfur lines difficult and, depending on the redshift, in many cases prevented these measurements, thus making 5800-6800 ̊ a compromise between these constrains.

Figure A.1 presents the spectra of the NLS1s for the red spectral region. All spectra are in rest frame for which we used the published redshift data from the NASA/IPAC Extragalactic Database (NED), and we present their fluxes in units of $10^{-17} \mathrm{erg} \mathrm{cm}^{-2} \mathrm{~s}^{-1} \AA^{-1}$. As expected from photoionized gas in active nuclei, $\mathrm{H} \alpha+[\mathrm{NII}]$ emission lines are the most important feature in all the objects, while in 25 out of 36 spectra $[\mathrm{SII}] \lambda \lambda 6716,6731$ lines are easily detected. We also identify [OI] $\lambda 6300$ (over $3 \sigma$ ) in only seven objects. Sodium lines $\mathrm{Na} \lambda \lambda 5890,5896$ were detected, although these (as well as other absorption lines) are often weak mainly because of the dilution of the stellar features by a nonstellar continuum; these lines also appear in emission in some cases.

1 IRAF: the Image Reduction and Analysis Facility is distributed by the National Optical Astronomy Observatories, which is operated by the Association of Universities for Research in Astronomy, Inc. (AURA) under cooperative agreement with the National Science Foundation (NSF). 

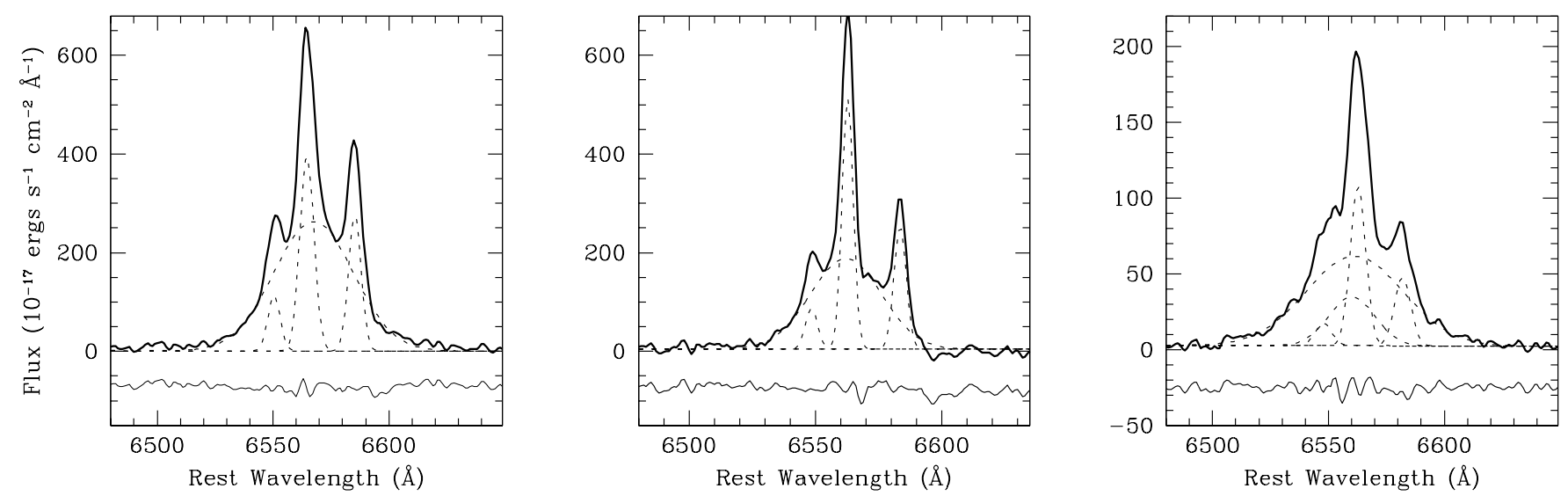

Fig. 1. From left to right: Gaussian decomposition for the galaxies MCG04.24.017, 2MASXJ05014863-2253232, and 2MASX J211244903730119 , respectively. Thick line represents the spectra; the $\mathrm{H} \alpha$ components and [NII] 26548,6584 are shown in dotted lines. The residuals are plotted at the bottom of each panel and were displaced from zero for clarity.

\section{Emission line measurements}

Our interest resides in the emission lines, which offer important information about kinematics and luminosities of the central regions of NLS1s. Emission line profiles of NLS1s can be represented by a single or a combination of Gaussian profiles. We used the LINER routine (Pogge \& Owen 1993), which is a $\chi^{2}$ minimization algorithm that can fit several Gaussians to a line profile. We adopted a procedure of fitting three possible Gaussians to $\mathrm{H} \alpha$ : one broad component (BC) to the wings of the line, usually extending to $\sim 5000 \mathrm{~km} \mathrm{~s}^{-1}$ in the base and with FWHM around $2500 \mathrm{~km} \mathrm{~s}^{-1}$; one narrow component (NC) fitted to the peak of the profile with typically $F W H M \sim 300 \mathrm{~km} \mathrm{~s}^{-1}$; and an intermediate component (IC) with FWHM between those of the NC and BC (IC is explained in detail in Sect. 4.3). It was not necessary to apply any Gaussian decomposition for the [NII] $\lambda \lambda 6548,6584$ and [SII] $\lambda \lambda 6716,6731$ lines, since one component for each line profile fits well. Two constraints were used for [NII] lines. First, [NII] $\lambda 6548$ and [NII] $\lambda 6584$ should be of similar FWHM because both lines are emitted in the same region and, second, the flux ratio should be equal to their theoretical value 1:3. Similar FWHM constraints as for [NII] were applied for [SII] lines. By this procedure, we obtained FWHM, center, and peak of each line, fully describing the Gaussians of $\mathrm{H} \alpha$, [NII], and [SII] lines. Typical uncertainties of the FWHM measurements are $\sim 10 \%$, while for the fluxes these are on the order of $\sim 15 \%$.

Figure 1 shows three typical fits for $\mathrm{H} \alpha+[\mathrm{NII}] \lambda 6548,6584$. Two of these are the same as the two components for $\mathrm{H} \alpha$ and one fit has the IC line. The solid thick line represents the spectra and the dotted lines represent each Gaussian profile fitted. The thin line represents the residuals of the fit (plotted below), which are similar to the noise level around the line. Figure 2 shows the distributions of the FWHM of $\mathrm{H} \alpha \mathrm{BC}, \mathrm{H} \alpha \mathrm{NC}$, [NII], and [SII]. All FWHM were corrected by the instrumental width as $F W H M^{2}=F W H M_{\text {obs }}^{2}-F W H M_{\text {inst }}^{2}$, where $F W H M_{\text {obs }}$ is the measured FWHM and $F W H M_{\text {inst }}$ is the instrumental broadening $\left(\sim 3.8 \AA\right.$ or $\left.170 \mathrm{~km} \mathrm{~s}^{-1}\right)$.

\section{Nuclear dynamics}

\subsection{Virial BH masses}

Black holes are very important in the study of active galaxies. They constitute a fundamental parameter to understand the
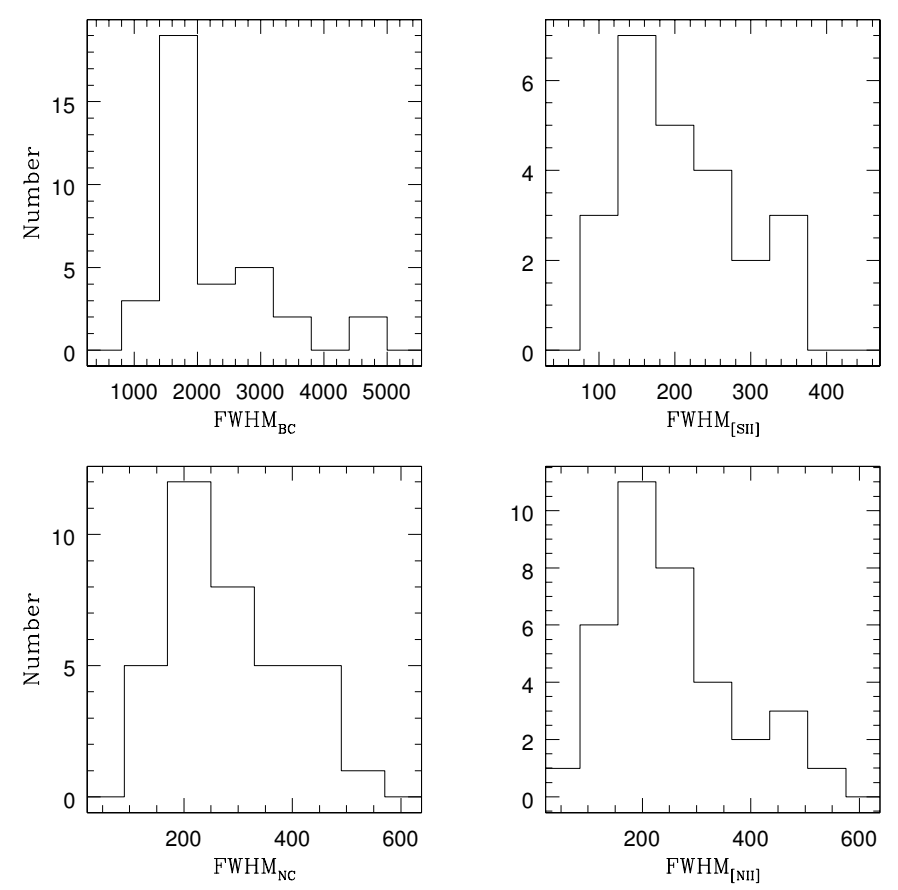

Fig. 2. Histograms of the FWHM of the emission lines in $\mathrm{km} \mathrm{s}^{-1}$. From left to right and top to bottom: $\mathrm{FWHM}$ of $\mathrm{BC},[\mathrm{SII}], \mathrm{NC}$ and $[\mathrm{NII}]$. All FWHM were corrected by the instrumental width.

mechanisms involved in the nuclear regions of AGNs and could also provide information about the process of galaxy formation. If we assume that the BLR clouds are isotropically spatially and kinematically distributed, $M_{\mathrm{BH}}$ is related to the radius of the $\operatorname{BLR}\left(R_{\mathrm{BLR}}\right)$ and the mean cloud velocity $v$ inside it as $M_{\mathrm{BH}}=$ $R_{\mathrm{BLR}} v^{2} G^{-1}$. According to Greene \& Ho (2005), BH masses can be calculated through luminosity and FWHM of the same Balmer line, as follows:

$M_{\mathrm{BH}}=2 \times 10^{6}\left(\frac{L_{\mathrm{H} \alpha}}{10^{42} \mathrm{erg} \mathrm{s}^{-1}}\right)^{0.55}\left(\frac{F W H M_{\mathrm{H} \alpha}}{10^{3} \mathrm{~km} \mathrm{~s}^{-1}}\right)^{2.06} M_{\odot}$.

This method is highly useful because Balmer lines are easily detectable even in distant AGNs. However, this equation can be applied only to AGNs that show broad component lines, thereby the importance of performing Gaussian deconvolution to Balmer lines such as described in Sect. 3. Using Eq. (1) we determined 


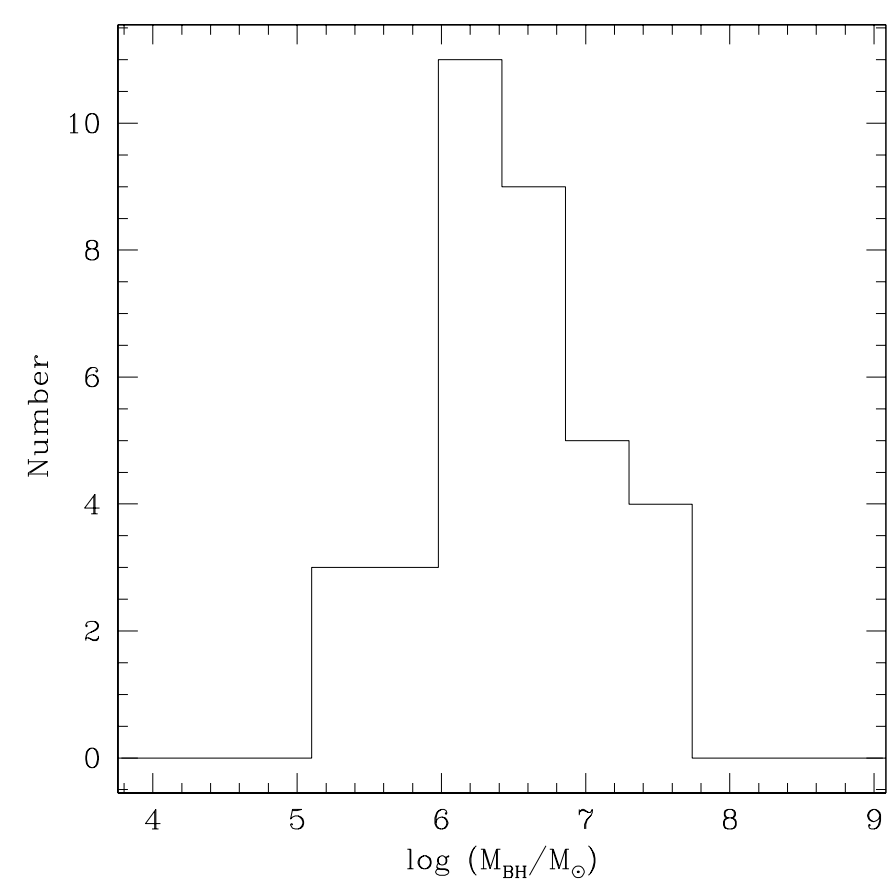

Fig. 3. Histogram of the black hole masses of the sample.

$M_{\mathrm{BH}}$ of our sample by taking into account the $\mathrm{BC}$ of $\mathrm{H} \alpha$ after correcting for instrumental resolution. Table A.2 specifies the galaxy name, FWHM, and luminosity of BC and $\mathrm{BH}$ masses. Figure 3 shows the distribution of $\mathrm{BH}$ masses, whose typical uncertainties are around $\sim 0.1$ dex. Almost all of the BH masses are between $\log \left(M_{\mathrm{BH}} / M_{\odot}\right) \sim 5.75$ and 7.35 (mean value $\sim 6.2$ ). Only two objects show masses $\log \left(M_{\mathrm{BH}} / M_{\odot}\right) \geq 7.5$; these objects are 6dF J1117042-290233, RX J2301.8-5508, which have $\log \left(M_{\mathrm{BH}} / M_{\odot}\right)$ of 7.7 and 7.5 , respectively. There are also a few determinations of low-mass BHs with $\log \left(M_{\mathrm{BH}} / M_{\odot}\right)<5.5$, mainly due to their low BC luminosities. These galaxies are SDSS J144052.60-023506.2, Zw374.029 with $\log \left(M_{\mathrm{BH}} / M_{\odot}\right)=$ 5.4 and NPM1G-17.0312 with $\log \left(M_{\mathrm{BH}} / M_{\odot}\right)=5.3$.

\subsection{The $\mathrm{M}_{\mathrm{BH}}-\sigma_{\star}$ relation}

Possible correlations between $\mathrm{BH}$ masses and properties of the host galaxies are of fundamental importance to understand the galaxy formation processes and evolution. The relations between black hole masses and the velocity dispersion of the bulge have been studied by several authors, such as Ferrarese \& Merritt (2000) and Gebhardt et al. (2000). Tremaine et al. (2002), in the attempt to relate some galaxy properties to central $\mathrm{BHs}$, found that $\mathrm{BH}$ masses for normal galaxies could be estimated with the relation,

$\log \left(\frac{M_{\mathrm{BH}}}{M_{\odot}}\right)=(8.13 \pm 0.06)+(4.02 \pm 0.32) \log \left(\frac{\sigma_{\star}}{200 \mathrm{~km} \mathrm{~s}^{-1}}\right)$.

On the other hand, in a sample of 14 Seyfert 1 galaxies, Nelson et al. (2004) measured the bulge stellar velocity dispersion and determined their SMBH masses using the reverberation mapping technique and found that the Seyfert galaxies followed the same $M_{\mathrm{BH}}-\sigma_{\star}$ relation as nonactive galaxies.

Usually it is difficult to determine the stellar velocity dispersions in AGNs because of the strong nonstellar continuum that dilutes the absorption lines of the underlying stellar populations. On the other hand, gas emission lines are an important feature in AGNs and they are detectable even in distant galaxies, unlike stellar absorption lines. Because of this, some authors (e.g., Nelson 2000; Komossa \& Xu 2007) use, for instance, [OIII] or [SII] lines instead the stellar velocity dispersion to study the $M_{\mathrm{BH}}-\sigma_{\star}$ relation. For example, when the width of the narrow [OIII] $\lambda 5007$ emission line is used, most authors found that in general NLS1 galaxies do not follow the $M_{\mathrm{BH}}-\sigma_{\star}$ relation (Grupe \& Mathur 2004; Bian et al. 2006; Watson et al. 2007). Nevertheless, Wang \& Lu (2001), Wandel (2002) found that NLS1 follow the relation but with a larger scatter than for normal galaxies.

In Fig. 4 we plot BH masses against $\mathrm{FWHM}_{\text {gas }}$, where the solid line represents $M_{\mathrm{BH}}-\sigma_{\star}$ relation for normal galaxies (Tremaine et al. 2002). We explore the $M_{\mathrm{BH}}-\sigma_{\star}$ relation using $\mathrm{FWHM}_{\text {gas }}$ instead of $\sigma_{\star}$, where $\mathrm{FWHM}_{\text {gas }}$ refers to the FWHM of the emission lines $\mathrm{NC}$ of $\mathrm{H} \alpha$, [NII], and [SII]. We take these widths as possible proxys of $\sigma_{\star}$, by taking into account the relation $F W H M=2.3548 \times \sigma$ for a Gaussian profile of these lines.

Taking the uncertainties of our determinations on $\mathrm{FWHM}_{\text {gas }}$ and black hole masses into account, Fig. 4 shows that, in general, most of the objects lie systematically below the $M_{\mathrm{BH}}-\sigma_{\star}$ relation for normal nuclei. In the case of [NII], around $70 \%$ of the galaxies lie below the Tremaine line. The higher deviation is seen using the $\mathrm{NC}$ of $\mathrm{H} \alpha$, where $\sim 80 \%$ of the objects lie below the $M_{\mathrm{BH}}-\sigma_{\star}$ relation. In the case of [SII] lines, NLS1s are closer to the relation and $\sim 45 \%$ of the targets lie above the relation. This agrees with previous results of Komossa \& Xu (2007). Despite the objects being closer to the Tremaine relation, they are slightly below it and we do not find any evidence of correlation among the FWHM of [SII] lines and the BH mass. The cases studied here of [SII], [NII], and NC lines are in agreement with the idea of that NLS1 may mainly reside in galaxies with pseudobulges (Mathur et al. 2012). In this scenario, NLS1 do not follow the $M_{\mathrm{BH}}-\sigma_{\star}$ because their bulges are intrinsically different from those of other galaxies.

Otherwise, the fact that, in general, NLS1 lie systematically below the $M_{\mathrm{BH}}-\sigma_{\star}$ relation for the three studied cases, could imply that NLS1 galaxies have lower BH masses compared to those that follow the relation. It is well known that NLS1 have high Eddington ratios (Warner et al. 2004) and related to that, Mathur (2000) proposed that NLS1 are analogous objects to high-redshift quasars $(z>4)$. This way, NLS1 may be in an early evolutionary phase that occupies young host galaxies. It is not very clear how the displacement of NLS1 would be along the $M_{\mathrm{BH}}-\sigma_{\star}$ relation, but the fact that NLS1 have high Eddington ratios suggest that their black hole masses must be rapidly growing (Mathur \& Grupe 2005a,b). In this scenario, the NLS1 tracks on the $M_{\mathrm{BH}}-\sigma_{\star}$ would be upward.

\subsection{The intermediate component of $\mathrm{H} \alpha$}

In Sect. 3 we mentioned that, besides the narrow and broad components for $\mathrm{H} \alpha$, for some objects it was necessary to include an additional kinematical intermediate component to fit the line. The sum of only two components (BC and NC) did not fit the profile of $\mathrm{H} \alpha$ well for some galaxies of our sample, thus giving residuals that were considerably higher than the spectral noise and requiring an additional component. Indeed, several components for the Balmer emission lines were detected in some AGNs (Popović et al. 2004; Zhu et al. 2009b) and NLS1s (Zhu et al. 2009a; Mullaney \& Ward 2008). While by definition BC and NC have a direct association with the BLR and NLR, respectively, described in the standard scenario for Seyferts, the IC remains clearly related to a well-known physical region. Various authors, 

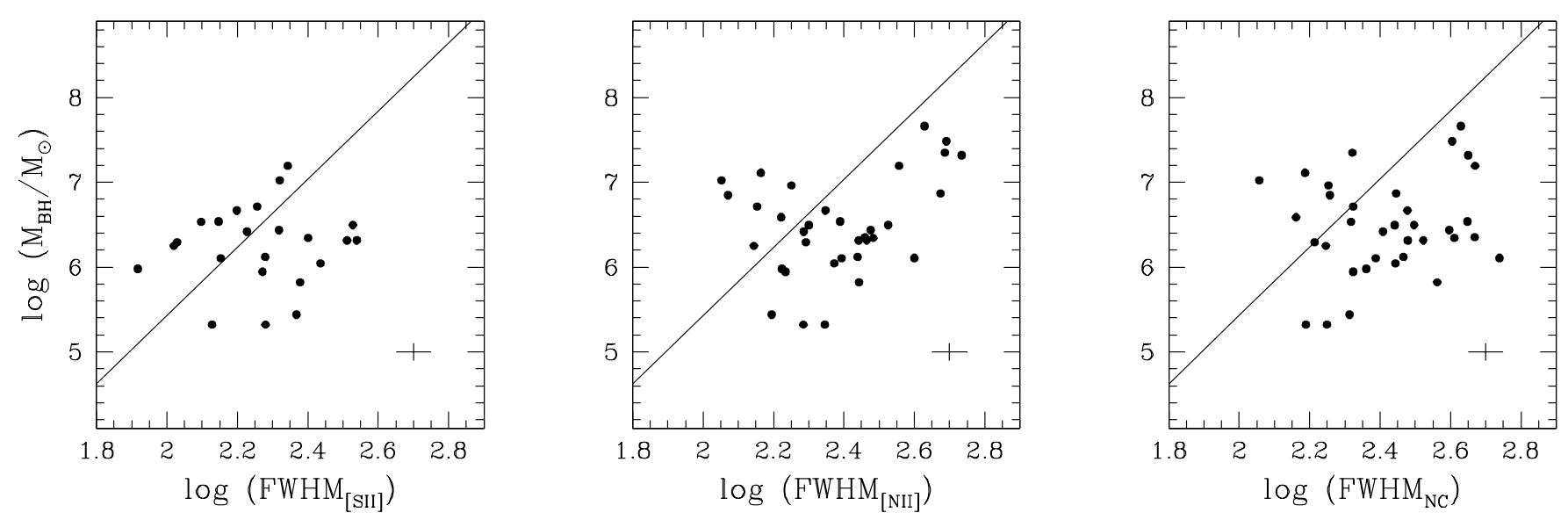

Fig. 4. $M_{\mathrm{BH}}-\sigma_{\star}$ relation for the galaxies of the sample, using the FWHM of [SII] (left), [NII] (middle), and the narrow component NC of H $\alpha$ (right). All FWHM are in $\mathrm{km} \mathrm{s}^{-1}$ and were corrected by the instrumental width. The solid line represents the relation given by Tremaine et al. using $F W H M=2.35 \sigma_{\star}$. Typical error bars are on each plot.

for example, Zhu et al. (2009a) and Zhu et al. (2009b), claimed that the emission from the BLR can be described by two Gaussian profiles, one very broad and an intermediate component. Both of these emissions could be originated in two different regions, each one with different velocity dispersions and spatially associated, but there is no clear scheme yet. To explore this kinematical component, we used our data from the IC detected in our sample.

Following the procedure described in Sect. 3 we determined the IC for 14 galaxies (37\% of the sample). The main results involving FWHM of $\mathrm{H} \alpha$ IC are presented in Fig. 5. The distribution of the FWHM (top left panel of Fig. 5) of IC shows that for 11 out of 14 galaxies, the FWHM of IC is in the range of $600-1500 \mathrm{~km} \mathrm{~s}^{-1}$ with mean value of $\sim 1100 \mathrm{~km} \mathrm{~s}^{-1}$. We note that the shape of the distributions for IC, BC, and NC are apparently similar. So, we have applied a Kolmogorov-Smirnov (K-S) test and confirmed that the distribution of the IC is significantly different from those of NC and BC with $P<10^{-8}$ and $P<10^{-5}$, respectively, as being drawn from the same parent distribution. This suggests the possibility of three kinematically distinct emitting regions: the well-known BLR and NLR, and an intermediate line region (ILR).

Comparing the $\mathrm{FWHM}_{\mathrm{IC}}$ and $\mathrm{FWHM}_{\mathrm{BC}}$ we found that they are strongly related (top right panel). An OLS bisector fit gives a slope of $\sim 2.4$ with a small $\left(<100 \mathrm{~km} \mathrm{~s}^{-1}\right)$ zero point and a remarkable Pearson correlation coefficient of $r_{\mathrm{p}}=0.93$. We calculated the ratio between the two quantities (bottom left panel), and we find a mean value of $F W H M_{\mathrm{BC}} / F W H M_{\mathrm{IC}} \sim 2.5$, which is somewhat lower than that obtained by Mao et al. (2010) of $\sim 3$ for $\mathrm{H} \beta$.

We have to keep in mind that the $\mathrm{BH}$ masses determinations (Eq. (1)) involves the BC, so we would expect a relation between the FWHM $\mathrm{IC}_{\mathrm{IC}}$ and the BH mass, which is actually seen in the bottom right panel of Fig. 5. An OLS bisector fit gives a slope of $\sim 4.4$ and a zero point of $\log \left(M_{\mathrm{BH}} / M_{\odot}\right) \sim-6.5$. A Pearson correlation coefficient of $r_{\mathrm{p}}=0.86$ shows the close relation between them, suggesting that the dynamics of the ILR is somehow affected by the central BH. We tested whether some of these relations depend on another parameter, such as the luminosity of the lines, but we did not find any dependence on them. A particular case is presented by the galaxy HE1107+0129, which presents an additional component of $\sim 290 \mathrm{~km} \mathrm{~s}^{-1}$ (see Sect. 6), and was not taken into account for the analysis in Fig. 5 and in the bottom right plot in Fig. 6. Besides this galaxy, IGRJ16185-5928 was not taken into account in the bottom right plot of Fig. 5 because of its high deviation from the remaining points.

The tight relationship between FWHM $_{\mathrm{BC}}$ and $\mathrm{FWHM}_{\mathrm{IC}}$ makes us wonder if there is another quantity governing this behavior. We do not have any information about the possible location of the origin of the IC. So far, we only know that, in the frame of the standard scenario for AGNs, BC and NC originate in the BLR and NLR regions, respectively; these two regions are very different in size, extension, geometry, kinematics, and physical conditions. The IC detected in our sample tells us that there could be another physical region. Under the assumption that the square of the FWHM of the gas decreases with the distance to the center, this ILR is possibly surrounding the BLR and could be located outside, although near the BLR with a ratio of mean sizes in a proportion given by the square of the ratio between the two FWHMs. This way the ratio of both would be $R_{\mathrm{ILR}} / R_{\mathrm{BLR}} \propto\left(F W H M_{\mathrm{BLR}} / F W H M_{\mathrm{ILR}}\right)^{2} \sim 4-9$.

\section{Luminosities}

We determined the luminosity for each component of $\mathrm{H} \alpha$, as well as for [NII] and [SII] lines. Figure 6 shows the luminosities of $\mathrm{BC}$ compared to those of NC (top left panel), [NII] $\lambda 6584$ (top right), [SII] 26731 (bottom left), and IC (bottom right).

The luminosities of $\mathrm{BC}$ are systematically higher than those of the lines originated at the NLR ([NII], [SII], and NC), and that of the IC, and a relation is evident between them. We found that the luminosity of the $\mathrm{BC}$ is typically eight times the luminosity of the NC, and only two objects (2MASXJ21565663-1139314 and SDSSJ144052.60-023506.2) present similar or lower luminosity of the BC compared to that of the NC. A similar trend is observed when comparing BC to [NII] $\lambda 6584$ and [SII] $\lambda 6731$. In the case of the IC, the luminosities of the $\mathrm{BC}$ are slightly higher for 10 out of 13 objects, and the remaining present weak BCs, and thus they have very low luminosities.

We applied OLS bisector fits to these data, which give slopes of around 1.2, 1.2, and 1.3 for NC, [NII], and [SII] lines, respectively. The Pearson coefficients are typically around $r_{\mathrm{p}}=0.67$ for these three relations. The slopes of the relations between the BC luminosity and that of the lines arising in the NLR are similar, which could suggest that the ionizing source is the same in the three cases. Interestingly, there is a stronger relationship between the BC and IC luminosities: the OLS bisector fit gives a slope of $\sim 0.84$ and a Pearson correlation coefficient of 0.90 , 

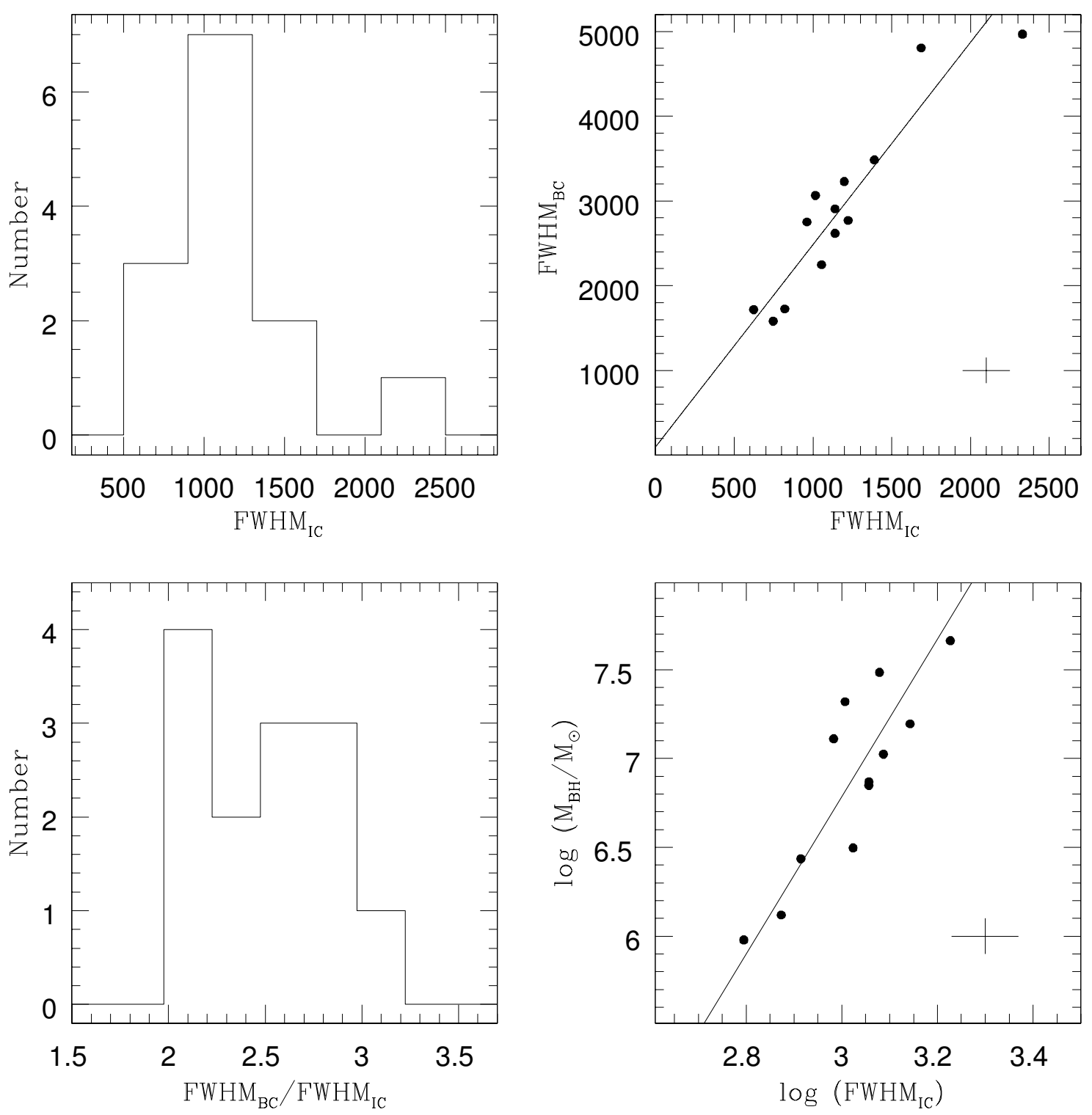

Fig. 5. Histogram of the FWHM of intermediate component (IC) of $\mathrm{H} \alpha$ (top left), the relation between the FWHM of BC and the FWHM of IC (top right), the histogram of $F W H M_{\mathrm{BC}} / F W H M_{\mathrm{IC}}$ (bottom left), and the relation between BH masses and the FWHM of IC (bottom right). All FWHM are in units of $\mathrm{km} \mathrm{s}^{-1}$. Typical error bars are shown. Solid lines represent the best fits for our data.

which probably implies a dependence among their emitting regions and also possibly on their physical conditions. The fact that for this case the slope is slower than that for narrow lines could suggest a possible physical difference between the emitting regions (ILR and NLR). Curiously, comparing $L_{\mathrm{BC}}$ to $L_{\mathrm{IC}}$, they are quite similar with a mean value of $L_{\mathrm{BC}} / L_{\mathrm{IC}} \sim 1.5$. This interesting result tells us that the luminosities of both regions are indeed comparable. The geometry of the emitting regions and the presence of different amount of gas in each region probably play an important role on their luminosities. Nonetheless, this is not the case for the ILR, for which, even though physical conditions are not well known yet, the relation between BC and IC is stronger than for NLR lines.

\section{Some curious cases}

Two objects show higher velocity dispersion for the IC. These galaxies are 6dF J1117042-290233 and IGRJ16185-5928, which have FWHM of $\sim 1700$ and $\sim 2000 \mathrm{~km} \mathrm{~s}^{-1}$, respectively. They also show the higher velocity dispersion for the $\mathrm{BC}, \sim 5000 \mathrm{~km} \mathrm{~s}^{-1}$, and the highest black hole masses, $\log \left(M_{\mathrm{BH}} / M_{\odot}\right)>7.3$.

We do not detect any $\mathrm{BC}$ for Zw037.022, which only has a NC for this Balmer line of $\sim 130 \mathrm{~km} \mathrm{~s}^{-1}$. According to Moran et al. (1996) and Kollatschny et al. (2008), this object has been classified as Sy1 and NLS1, respectively. On the other hand, according to the spectral classification of the Sloan Digital Sky Survey Data Release 12 (SDSS DR12; Alam et al. 2015; Eisenstein et al. 2011), Zw037.022 is a star forming galaxy. This classification is based on whether the galaxy has detectable emission lines that are consistent with star formation according to the criteria $\log ([\mathrm{OIII}] \lambda 500 / \mathrm{H} \alpha)<$ $0.7-1.2(\log ([\mathrm{NII}] \lambda 6584 / \mathrm{H} \alpha)+0.4)$. In our case, we find that $\log ([\mathrm{NII}] \lambda 6584 / \mathrm{H} \alpha)=-0.8, \log ([\mathrm{SII}] \lambda 6716 / \mathrm{H} \alpha)=-0.8$, and $\log ([\mathrm{SII}] \lambda 6731 / \mathrm{H} \alpha)=-0.9$. These results agree with the idea of that this object must be classified as a star forming galaxy. In 

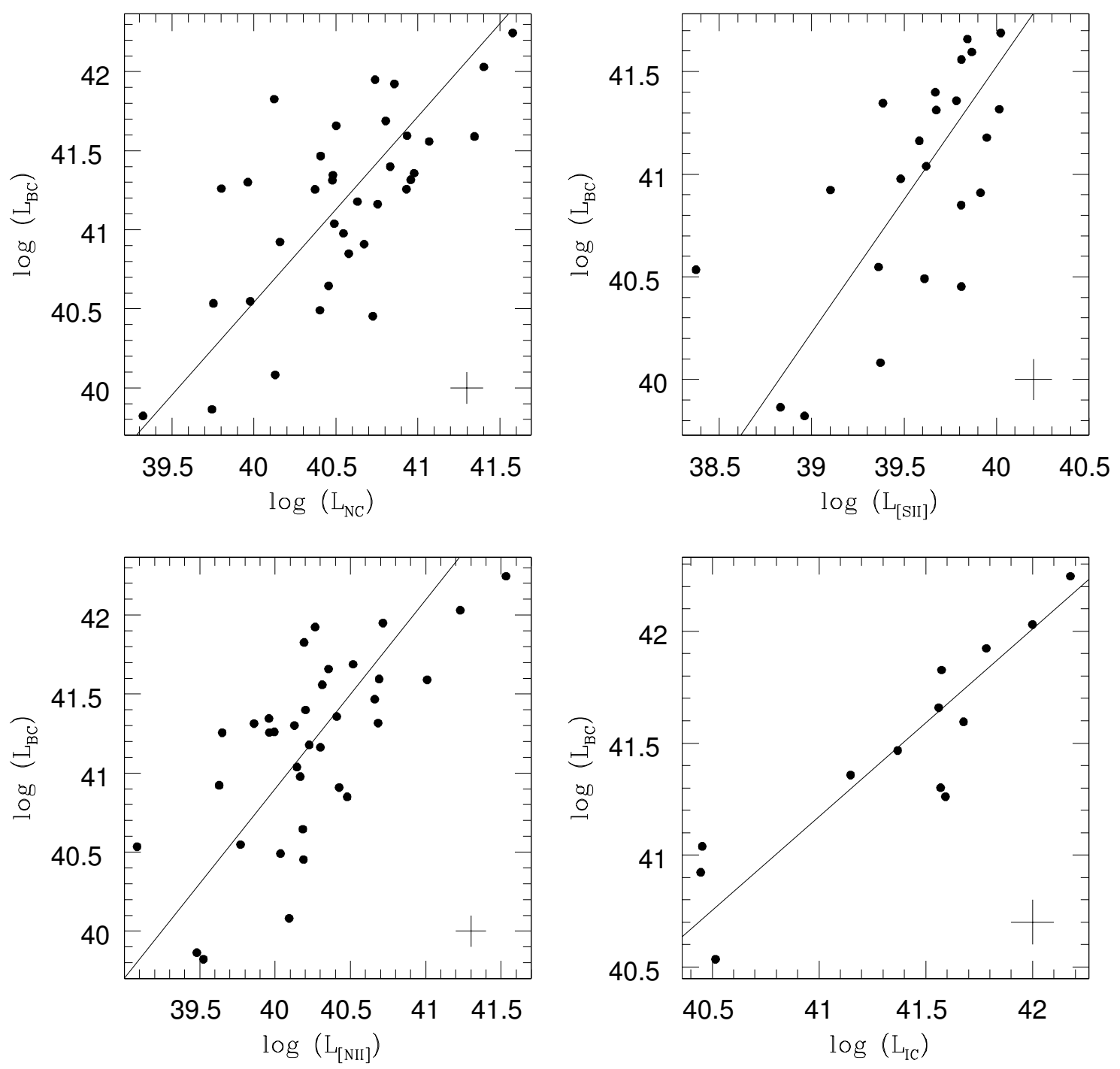

Fig. 6. Luminosity of BC compared with those of the NLR: NC (top left), [SII] (top right), [NII] (bottom left), and with the IC (bottom right). All luminosities are in units of $\mathrm{erg} \mathrm{s}^{-1}$. Solid lines represent the best fits for our data. Typical error bars are shown in each plot.

addition, the fact that this galaxy does not show a BC supports the idea that it is neither a Sy1 nor a NLS1 galaxy.

As stated in Sect. 4.3, the object HE1107+0129 shows an additional component of $\sim 290 \mathrm{~km} \mathrm{~s}^{-1}$. We tried to fit the spectrum of this galaxy with only two components for $\mathrm{H} \alpha$, but the residuals were much higher than the noise of the spectrum, and so another component was necessary. This extra component is comparable with the FWHM distribution of NC and shows a blueshift of $\sim 360 \mathrm{~km} \mathrm{~s}^{-1}$ compared to the NC (FWHM of $\sim 190 \mathrm{~km} \mathrm{~s}^{-1}$ ). We analyzed the relation between the FWHM of BC and NC for the sample and the medium value is $F W H M_{\mathrm{BC}} / F W H M_{\mathrm{NC}} \sim 8$. For this galaxy, the ratio between the FWHM $\mathrm{BC}$ and the FWHM of this additional component is also $\sim 8$. These facts suggest that this extra component is emitted from the NLR and not from an ILR. For this reason, it was not taken into account in the analysis of Sect. 4.3.

\section{Final remarks}

We have observed and analyzed the spectroscopic data of a sample of 36 NLS1 galaxies, 27 of which are in the Southern Hemisphere. We performed careful Gaussian decomposition to the main emission lines in the red spectral range. Several components were fitted to the global profile for $\mathrm{H} \alpha+[\mathrm{NII}] \lambda \lambda 6548,6583$. This allowed us to estimate the virial $\mathrm{BH}$ masses of the galaxies. The obtained values for our sample are in the range $\log \left(M_{\mathrm{BH}} / M_{\odot}\right)=5.3-7.7$, where the mean value is around $\log \left(M_{\mathrm{BH}} / M_{\odot}\right)=6.2$. The black hole masses are in the range $\log \left(M_{\mathrm{BH}} / M_{\odot}\right)=5.7-7.3$ for 32 out of 36 galaxies. These values are lower that those found in broad line Seyfert 1 galaxies, confirming that on average, NLS1 have lower BH masses (e.g., Komossa \& Xu 2007; Grupe 2004).

We tested the narrow component of $\mathrm{H} \alpha$, [NII], and [SII] lines as proxys of $\sigma_{\star}$, using the FWHM of these emission lines to examine the $M_{\mathrm{BH}}-\sigma_{\star}$ relation for normal galaxies. Taking into account the uncertainties of our determinations on the FWHM of the emission lines and black hole masses, we found that in general most of our NLS1s lie below the $M_{\mathrm{BH}}-\sigma_{\star}$ relation for normal galaxies. The fact that NLS1 have lower black hole masses, and taking into account that they have high acretion rates (Warner et al. 2004) and therefore their black hole mass are growing quickly (Mathur \& Grupe 2005a,b), could suggest that such AGNs are at an early stage of nuclear activity. Although in the case of the [SII] lines the galaxies seem to be closer to the relation than the other lines (in agreement with Komossa \& Xu 2007, results), we do not see any correlation between the FWHM 
of [SII] lines and the BH mass. The fact that the three tested emission lines put the NLS1 below the $M_{\mathrm{BH}}-\sigma_{\star}$ relation could be in agreement with Mathur et al. (2012), who found that such AGNs are mainly hosted by galaxies with pseudobulges. Related to this, NLS1 would not follow the $M_{\mathrm{BH}}-\sigma_{\star}$ because their bulges are different from those of other AGN types.

We studied the intermediate component of $\mathrm{H} \alpha$ found in 13 out of 36 galaxies. Comparing the distribution of velocities between the three components of $\mathrm{H} \alpha$, we see that the FWHM of BC is between $900-3200 \mathrm{~km} \mathrm{~s}^{-1}$ with a peak in $1600 \mathrm{~km} \mathrm{~s}^{-1}$ for most objects; the IC is in the velocity range $700-1500 \mathrm{~km} \mathrm{~s}^{-1}$ for most galaxies, with most objects showing FWHM of $1100 \mathrm{~km} \mathrm{~s}^{-1}$; while for the NC the velocity range is $180-500 \mathrm{~km} \mathrm{~s}^{-1}$ with a peak in $220 \mathrm{~km} \mathrm{~s}^{-1}$. We note a high correlation between $\mathrm{FWHM}_{\mathrm{IC}}$ and $\mathrm{FWHM}_{\mathrm{BC}}$, with a Pearson correlation coefficient of $r_{\mathrm{p}}=0.93$. We found for our sample that $F W H M_{\mathrm{BC}} / F W H M_{\mathrm{IC}}$ is in a range of $2.1-3$ with a mean value of $\sim 2.5$, which is somewhat lower than that obtained by Mao et al. (2010) of $\sim 3$ for $\mathrm{H} \beta$. We also performed Kolmogorov-Smirnov $(\mathrm{K}-\mathrm{S})$ tests to $\mathrm{FWHM}_{\mathrm{BC}}$ and $\mathrm{FWHM}_{\mathrm{IC}}$, and to $\mathrm{FWHM}_{N C}$ and FWHM $_{\mathrm{IC}}$, which give probabilities of $P<10^{-5}$ and $P<10^{-8}$ as being drawn from the same parent distribution, respectively. All these results point out the possibility of three kinematically distinct emitting regions and show that $\mathrm{BC}$ and IC are somehow linked, possibly implying that IC could arise in a region surrounding the BLR. Taking into account that $\mathrm{BH}$ masses depend on $\mathrm{FWHM}_{\mathrm{BC}}$, and $\mathrm{FWHM}_{\mathrm{IC}}$ correlates with $\mathrm{FWHM}_{\mathrm{BC}}$, it is expected to find a correlation between $\mathrm{BH}$ masses and $\mathrm{FWHM}_{\mathrm{IC}}$. In fact, this correlation has a Pearson correlation coefficient $r_{\mathrm{p}}=0.86$, suggesting that the dynamics of this emitting region is clearly affected by the central engine.

The presence of the additional intermediate component mainly affects the NC. If only two components were used to fit the $\mathrm{H} \alpha$ line, NC would be much broader and would show a greater amount of line flux. In the case of the $\mathrm{BC}$, it does not vary significantly; in general it could increase its FWHM by $\sim 10 \%$ and decrease the flux by $\sim 10-25 \%$. Contrary to what we expected, BH masses in general decrease $\sim 10-20 \%$ if one consider only two components for this Balmer line.

We also studied the emission line luminosities of broad and narrow lines. There are some tendencies between the BC luminosity and those of the narrow lines [NII], [SII], and NC with Pearson correlation coefficients around 0.67 for the three cases, indicating that the emission in BLR and NLR are proportional. Interestingly, their slopes are similar, around 1.2 , as derived from OLS bisector fits. The relation is even stronger for the IC with a slope of around 0.84 and a Pearson correlation coefficient of 0.90 . The fact that the slope is different for the NC and the IC lines would indicate possible physical difference between the regions. Interestingly, we found that the luminosities of $\mathrm{BC}$ and IC are comparable with a mean value $L_{\mathrm{BC}} / L_{\mathrm{IC}} \sim 1.5$. Related to this, the geometry of the emitting regions, despite distinct amounts of gas present in each one, play an important role. Even though the physical conditions of the ILR are not well known yet, our results suggest that this region is different from the BLR and NLR, with properties that are somehow related and strongly linked to those of the BLR.

Acknowledgements. This work was partially supported by Consejo de Investigaciones Científicas y Técnicas (CONICET) and Secretaría de Ciencia y Técnica de la Universidad Nacional de Córdoba (SecyT). We want to thank the anonymous referee, whose very useful remarks helped us to improve this paper. This research has made use of the NASA/IPAC Extragalactic Database (NED) which is operated by the Jet Propulsion Laboratory, California Institute of Technology, under contract with the National Aeronautics and Space Administration.

\section{References}

Alam, S., Albareti, F. D., Allende Prieto, C., et al. 2015, ApJS, 219, 12 Bian, W., \& Zhao, Y. 2004, MNRAS, 347, 607

Bian, W., Yuan, Q., \& Zhao, Y. 2006, MNRAS, 367, 860

Botte, V., Ciroi, S., di Mille, F., Rafanelli, P., \& Romano, A. 2005, MNRAS, 356, 789

Brotherton, M. S., Wills, B. J., Francis, P. J., \& Steidel, C. C. 1994, ApJ, 430, 495

Corbin, M. R., \& Francis, P. J. 1994, AJ, 108, 2016

Crenshaw, D. M., \& Kraemer, S. B. 2007, ApJ, 659, 250

Crenshaw, D. M., Kraemer, S. B., Schmitt, H. R., et al. 2009, ApJ, 698, 281

Eisenstein, D. J., Weinberg, D. H., Agol, E., et al. 2011, AJ, 142, 72

Ferrarese, L., \& Merritt, D. 2000, ApJ, 539, L9

Gebhardt, K., Bender, R., Bower, G., et al. 2000, ApJ, 539, L13

Goodrich, R. W. 1989, ApJ, 342, 224

Greene, J. E., \& Ho, L. C. 2005, ApJ, 630, 122

Grupe, D. 2004, AJ, 127, 1799

Grupe, D., \& Mathur, S. 2004, ApJ, 606, L41

Hu, C., Wang, J.-M., Ho, L. C., et al. 2008, ApJ, 683, L115

Kollatschny, W., Kotulla, R., Pietsch, W., Bischoff, K., \& Zetzl, M. 2008, A\&A, 484,897

Komossa, S., \& Xu, D. 2007, ApJ, 667, L33

Mao, W., Hu, C., Wang, J., et al. 2010, Science China Physics, Mechanics, and Astronomy, 53, 2307

Mason, K. O., Puchnarewicz, E. M., \& Jones, L. R. 1996, MNRAS, 283, L26

Mathur, S. 2000, MNRAS, 314, L17

Mathur, S., \& Grupe, D. 2005a, A\&A, 432, 463

Mathur, S., \& Grupe, D. 2005b, ApJ, 633, 688

Mathur, S., Kuraszkiewicz, J., \& Czerny, B. 2001, New Astron., 6, 321

Mathur, S., Fields, D., Peterson, B., \& Grupe, D. 2012, ApJ, 754, 146

Moran, E. C., Halpern, J. P., \& Helfand, D. J. 1996, ApJS, 106, 341

Mullaney, J. R., \& Ward, M. J. 2008, MNRAS, 385, 53

Nelson, C. H. 2000, ApJ, 544, L91

Nelson, C. H., Green, R. F., Bower, G., Gebhardt, K., \& Weistrop, D. 2004, ApJ, 615,652

Osterbrock, D. E., \& Pogge, R. W. 1985, ApJ, 297, 166

Pogge, R. W., \& Owen, J. M. 1993, OSU Internal Report, 93-01

Popović, L. Č., Mediavilla, E., Bon, E., \& Ilić, D. 2004, A\&A, 423, 909

Tremaine, S., Gebhardt, K., Bender, R., et al. 2002, ApJ, 574, 740

Véron-Cetty, M.-P., \& Véron, P. 2010, A\&A, 518, A10

Wandel, A. 2002, ApJ, 565, 762

Wang, T., \& Lu, Y. 2001, A\&A, 377, 52

Warner, C., Hamann, F., \& Dietrich, M. 2004, ApJ, 608, 136

Watson, L. C., Mathur, S., \& Grupe, D. 2007, AJ, 133, 2435

Williams, R. J., Pogge, R. W., \& Mathur, S. 2002, AJ, 124, 3042

Zhou, H., Wang, T., Yuan, W., et al. 2006, ApJS, 166, 128

Zhu, L., Zhang, S., \& Tang, S. 2009a, ArXiv e-prints [arXiv: 0901.2167]

Zhu, L., Zhang, S. N., \& Tang, S. 2009b, ApJ, 700, 1173 


\section{Appendix A: Additional data}

Table A.1. Observed galaxies.

\begin{tabular}{|c|c|c|c|c|c|c|}
\hline Galaxy & $\begin{array}{c}\text { RA } \\
\mathrm{J}(2000)\end{array}$ & $\begin{array}{c}\text { Dec } \\
\mathrm{J}(2000)\end{array}$ & $m_{\mathrm{b}}$ & $z$ & Date & $\begin{array}{c}\text { Exposure time } \\
\text { (s) }\end{array}$ \\
\hline 1RXS J040443.5-295316 & 040443.1 & -295323 & 17.3 & 0.06002 & $08-09-2013$ & $3 \times 2400$ \\
\hline 2MASXJ01115114-4045426 & 011151.1 & -404543 & 16.9 & 0.05429 & $11-01-2011$ & $3 \times 1800$ \\
\hline 2MASX J01413249-1528016 & 014132.5 & -152801 & 16.9 & 0.08106 & $10-27-2014$ & $3 \times 1800$ \\
\hline 2MASXJ05014863-2253232 & 050148.6 & -225323 & 14.0 & 0.04080 & $10-29-2011$ & $3 \times 1800$ \\
\hline 2MASX J21124490-3730119 & 211244.9 & -373012 & 17.0 & 0.04297 & $08-17-2012$ & $3 \times 1800$ \\
\hline 2MASX J21531910-1514111 & 215319.1 & -151412 & 14.7 & 0.07780 & $10-25-2014$ & $2 \times 2400$ \\
\hline 2MASXJ21565663-1139314 & 215656.5 & -113932 & 15.4 & 0.02808 & $09-07-2013$ & $3 \times 2400$ \\
\hline 6dF J1117042-290233 & 111704.2 & -290233 & 15.8 & 0.07046 & $04-26-2013$ & $4 \times 1800$ \\
\hline CTSJ13.12 & 135129.5 & -181347 & 17.5 & 0.01221 & $04-23-2012$ & $3 \times 1800$ \\
\hline CTSM02.47 & 104623.5 & -300420 & 17.0 & 0.05706 & $04-25-2012$ & $3 \times 1800$ \\
\hline EUVEJ0349-53.7 & 034928.5 & -534447 & 15.7 & 0.13000 & $10-27-2014$ & $3 \times 2400$ \\
\hline FAIRALL0107 & 213529.5 & -623007 & 16.7 & 0.06096 & $01-11-2011$ & $3 \times 1800$ \\
\hline HE1107+0129 & 111012.1 & +011327 & 16.5 & 0.09550 & 09-04-2013 & $2 \times 2400$ \\
\hline HE1438-0159 & 144111.5 & -021235 & 16.6 & 0.08292 & $02-05-2014$ & $3 \times 2400$ \\
\hline IGRJ16185-5928 & 161836.4 & -592718 & 16.5 & 0.03462 & $04-25-2012$ & $3 \times 1800$ \\
\hline IRAS04576+0912 & 050020.8 & +091656 & 16.6 & 0.03609 & $01-11-2011$ & $2 \times 1800$ \\
\hline IRAS16355-2049 & 163830.9 & -205525 & 14.5 & 0.02637 & $08-15-2012$ & $3 \times 1800$ \\
\hline MCG-04.24.017 & 105555.4 & -230325 & 14.5 & 0.01281 & $04-24-2012$ & $3 \times 2400$ \\
\hline MCG-05.01.013 & 235327.9 & -302740 & 15.8 & 0.03068 & $09-09-2013$ & $3 \times 2400$ \\
\hline NPM1G-17.0312 & 114042.2 & -174010 & 15.9 & 0.02187 & $04-23-2012$ & $4 \times 1800$ \\
\hline RBS1665 & 200015.5 & -541712 & 16.5 & 0.06070 & $04-25-2012$ & $3 \times 1800$ \\
\hline RX J0024.7+0820 & 002445.7 & +082057 & 17.2 & 0.06700 & $10-25-2014$ & $3 \times 2400$ \\
\hline RX J0323.2-4931 & 032315.3 & -493107 & 16.5 & 0.07100 & $10-29-2011$ & $3 \times 1800$ \\
\hline RX J0902.5-0700 & 090233.6 & -070004 & 17.7 & 0.08911 & $04-09-2013$ & $2 \times 2400$ \\
\hline RX J2301.8-5508 & 230152.0 & -550831 & 14.7 & 0.14100 & $10-27-2014$ & $3 \times 2400$ \\
\hline SDSS J134524.69-025939.8 & 134524.7 & -025940 & 17.1 & 0.08552 & $04-09-2013$ & $3 \times 2400$ \\
\hline SDSS J144052.60-023506.2 & 144052.6 & -023506 & 16.3 & 0.04434 & $04-30-2014$ & $3 \times 2400$ \\
\hline SDSS J151024.92+005843.9 & 151024.9 & +005844 & 17.3 & 0.07215 & $04-06-2013$ & $2 \times 1800$ \\
\hline SDSS J153001.82-020415.1 & 153001.8 & -020415 & 17.1 & 0.05118 & $05-02-2014$ & $3 \times 2400$ \\
\hline SDSS J153705.95+005522.8 & 153705.9 & +005523 & 17.3 & 0.13655 & $04-09-2013$ & $3 \times 2400$ \\
\hline SDSS J225452.22+004631.4 & 225452.2 & +004631 & 17.4 & 0.09073 & $08-09-2013$ & $3 \times 2400$ \\
\hline V961349-439 & 135259.6 & -441325 & 15.4 & 0.05200 & $04-06-2013$ & $2 \times 1800$ \\
\hline WPV8507 & 003915.8 & -511701 & 15.8 & 0.02861 & $08-18-2012$ & $3 \times 1800$ \\
\hline Zw037.022 & 102359.8 & +062907 & 16.0 & 0.04385 & $04-30-2014$ & $3 \times 2400$ \\
\hline Zw049.106 & 151751.7 & +050628 & 17.5 & 0.03878 & $04-30-2014$ & $3 \times 2400$ \\
\hline Zw374.029 & 205522.3 & +022116 & 15.1 & 0.01356 & $04-23-2012$ & $2 \times 1800$ \\
\hline
\end{tabular}

Notes. Column 1: galaxy name. Columns 2 to 5: right ascension (J2000), declination (J2000), apparent B magnitude, and redshift, as taken from NED. Columns 6 and 7: observation date and exposure time. 
Table A.2. FWHM of the $\mathrm{BC}$ of $\mathrm{H} \alpha$, luminosity of that component, and $\mathrm{BH}$ masses.

\begin{tabular}{|c|c|c|c|}
\hline Galaxy & $\begin{array}{l}F W H M_{\mathrm{BC}} \\
\left(\mathrm{km} \mathrm{s}^{-1}\right)\end{array}$ & $\begin{array}{l}\log L_{\mathrm{BC}} \\
\left(\operatorname{erg~s}^{-1}\right)\end{array}$ & $\begin{array}{l}\log M_{\mathrm{BH}} \\
\left(M_{\odot}\right)\end{array}$ \\
\hline 1RXS J040443.5-295316 & 1848 & 40.91 & 6.2 \\
\hline 2MASXJ01115114-4045426 & 1741 & 41.31 & 6.4 \\
\hline 2MASX J01413249-1528016 & 1518 & 41.4 & 6.3 \\
\hline 2MASXJ05014863-2253232 & 1510 & 41.32 & 6.3 \\
\hline 2MASX J21124490-3730119 & 2248 & 41.04 & 6.5 \\
\hline 2MASX J21531910-1514111 & 1852 & 40.65 & 6.1 \\
\hline 2MASXJ21565663-1139314 & 1903 & 40.08 & 5.8 \\
\hline 6dF J1117042-290233 & 4806 & 41.92 & 7.7 \\
\hline CTSJ13.12 & 1717 & 40.53 & 6.0 \\
\hline CTSM02.47 & 1964 & 41.26 & 6.5 \\
\hline EUVEJ0349-537 & 3065 & 42.03 & 7.3 \\
\hline FAIRALL0107 & 1709 & 41.56 & 6.5 \\
\hline HE1107+0129 & 2166 & 41.95 & 6.9 \\
\hline HE1438-0159 & 1673 & 41.26 & 6.3 \\
\hline IGRJ16185-5928 & 4966 & 41.3 & 7.3 \\
\hline IRAS04576+0912 & 1685 & 41.18 & 6.3 \\
\hline IRAS16355-2049 & 3483 & 41.6 & 7.2 \\
\hline MCG-04.24.017 & 1835 & 40.55 & 6.0 \\
\hline MCG-05.01.013 & 1939 & 41.35 & 6.5 \\
\hline NPM1G-17.0312 & 1243 & 39.86 & 5.3 \\
\hline RBS1665 & 1725 & 41.36 & 6.4 \\
\hline RX J0024.7+0820 & 1504 & 40.98 & 6.1 \\
\hline RX J0323.2-4931 & 2752 & 41.83 & 7.1 \\
\hline RX J0902.5-0700 & 2770 & 41.66 & 7.0 \\
\hline RX J2301.8-5508 & 3228 & 42.25 & 7.5 \\
\hline SDSS J134524.69-025939.8 & 1925 & 41.69 & 6.7 \\
\hline SDSS J144052.60-023506.2 & 987.4 & 40.45 & 5.4 \\
\hline SDSS J151024.92+005843.9 & 2522 & 41.16 & 6.7 \\
\hline SDSS J153001.82-020415.1 & 1699 & 40.49 & 6.0 \\
\hline SDSS J153705.95+005522.8 & 1774 & 41.59 & 6.6 \\
\hline SDSS J225452.22+004631.4 & 2617 & 41.47 & 6.9 \\
\hline V961349-439 & 2906 & 41.26 & 6.8 \\
\hline WPV85007 & 1582 & 40.92 & 6.1 \\
\hline Zw037.022 & - & - & - \\
\hline Zw049.106 & 2060 & 40.85 & 6.3 \\
\hline Zw374.029 & 1276 & 39.82 & 5.4 \\
\hline
\end{tabular}

Notes. The galaxy Zw037.022 does not show the broad component (see Sect. 6). 
E. O. Schmidt et al.: Spectral nuclear properties of NLS1 galaxies
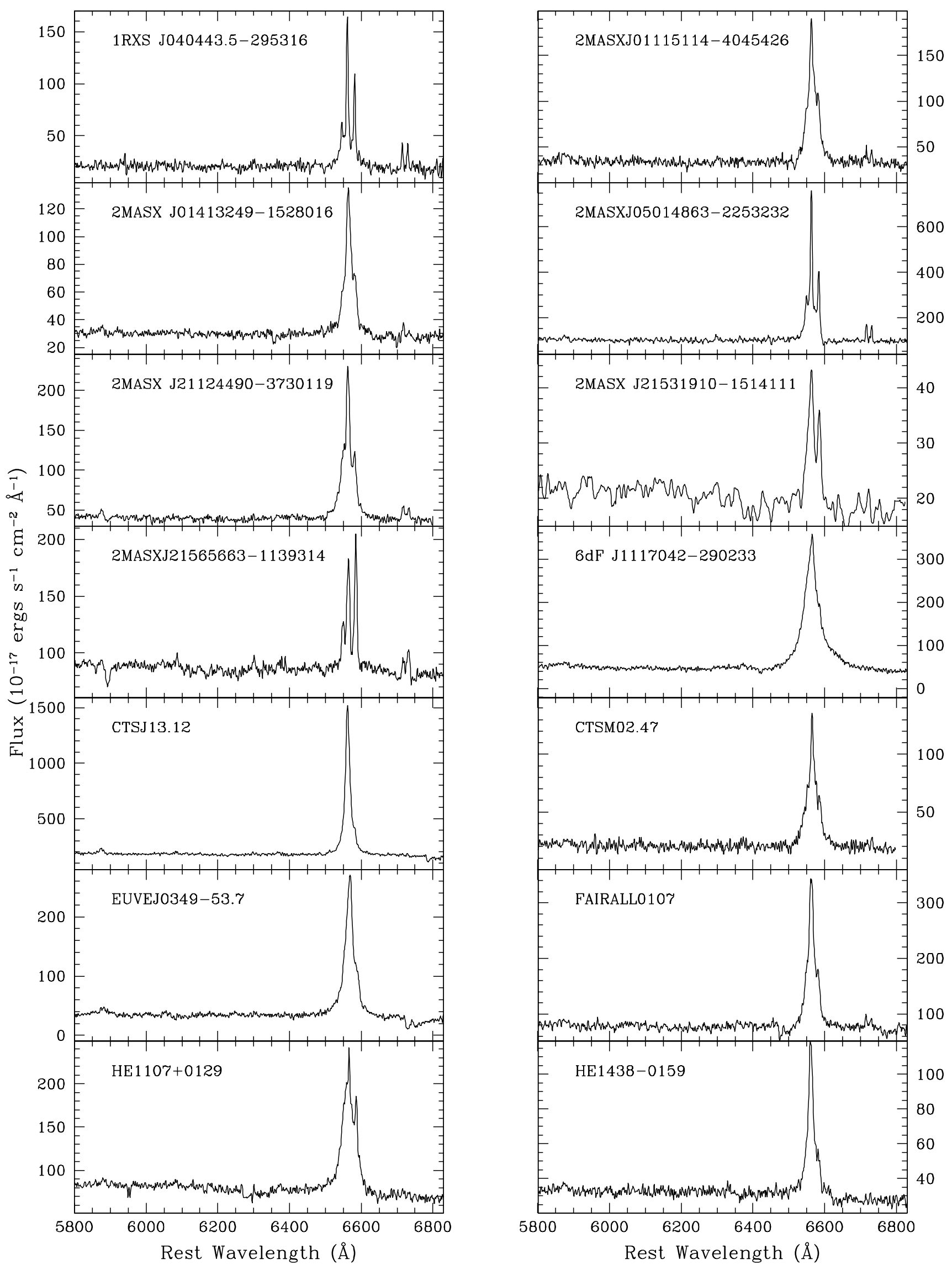

Fig. A.1. Observed spectra of the NLS1 in the range 5800-6800 $\AA$ at rest frame. 

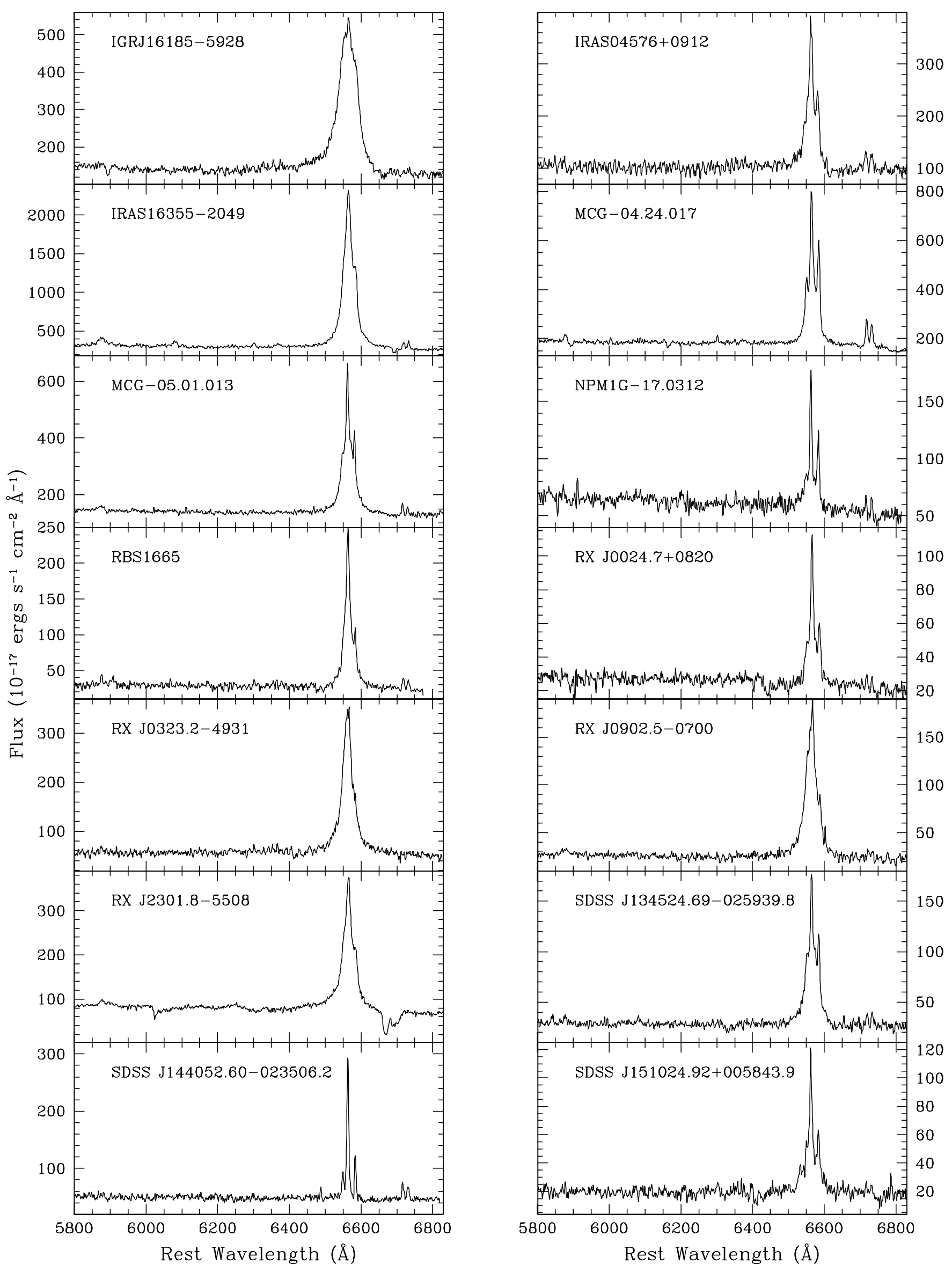

Fig. A.1. continued. 
E. O. Schmidt et al.: Spectral nuclear properties of NLS1 galaxies
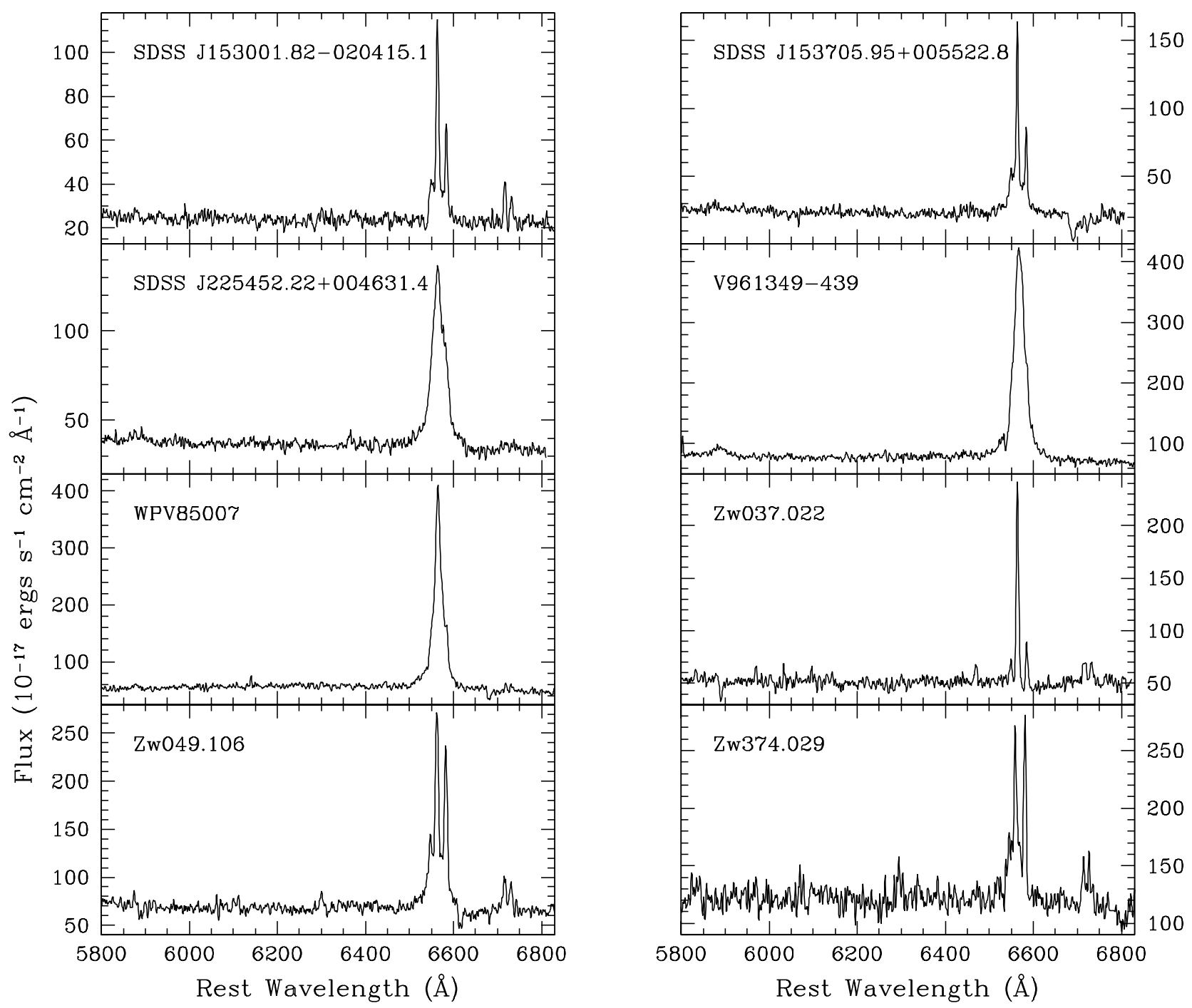

Fig. A.1. continued. 